Wilfrid Laurier University

Scholars Commons @ Laurier

Lyle S. Hallman Social Work Faculty

Publications

Lyle S. Hallman Faculty of Social Work

$12-15-2015$

\title{
A comparison of walk-in counselling and the wait list model for delivering counselling services
}

\author{
Carol Stalker \\ Wilfrid Laurier University, cstalker@wlu.ca \\ Manuel Riemer \\ Wilfrid Laurier University \\ Cheryl-Anne Cait \\ Wilfrid Laurier University \\ Susan Horton \\ University of Waterloo \\ Jocelyn Booton \\ Wilfrid Laurier University
}

See next page for additional authors

Follow this and additional works at: https://scholars.wlu.ca/scwk_faculty

Part of the Social Work Commons

\section{Recommended Citation}

Stalker C.A., Riemer, M., Cait, C.A., Horton, S., Booton, J., Josling, L., Bedggood, J. \& Zaczek M. (2015). A comparison of walk-in counselling and the wait list model for delivering counselling services. Journal of Mental Health. Published on-line: 15 December 2015. DOI:10.3109/09638237.2015.1101417. This study was funded by Canadian Institutes of Health Research (CIHR) FRN 119528.

This Article is brought to you for free and open access by the Lyle S. Hallman Faculty of Social Work at Scholars Commons@ Laurier. It has been accepted for inclusion in Lyle S. Hallman Social Work Faculty Publications by an authorized administrator of Scholars Commons @ Laurier. For more information, please contact scholarscommons@wlu.ca. 


\section{Authors}

Carol Stalker, Manuel Riemer, Cheryl-Anne Cait, Susan Horton, Jocelyn Booton, Leslie Josling, Joanna Bedggood, and Margaret Zaczek 
This is the pre-peer-reviewed version of the following article:

Stalker C.A., Riemer, M., Cait, C.A., Horton, S., Booton, J., Josling, L., Bedggood, J. \& Zaczek M. (2015). A comparison of walk-in counselling and the wait list model for delivering counselling services. Journal of Mental Health. DOI:10.3109/09638237.2015.1101417, which was published on-line on December 15, 2015. See http://www.tandfonline.com/doi/full/10.3109/09638237.2015.1101417

This study was funded by Canadian Institutes of Health Research (CIHR) FRN 119528. 
WALK-IN COUNSELLING VS TRADITIONAL

Running head: WALK-IN COUNSELLING VS TRADITIONAL

\section{A comparison of single-session walk-in counselling and the traditional model for delivering counselling services}

\section{Carol A. Stalker ${ }^{1}$, Manuel Riemer ${ }^{2}$, Cheryl-Anne Cait ${ }^{3}$, Susan Horton ${ }^{4}$, Jocelyn Booton ${ }^{5}$, Leslie Josling 6 , Joanna Bedggood ${ }^{7}$ and Margaret Zaczek $^{8}$}

${ }^{1}$ Professor, Faculty of Social Work, Wilfrid Laurier University

2 Associate Professor, Department of Psychology, Wilfrid Laurier University

${ }^{3}$ Associate Professor, Faculty of Social Work, Wilfrid Laurier University

4 Professor, Centre for International Governance Innovation, Chair in Global Health

Economics, University of Waterloo

5 Research Coordinator, Faculty of Social Work, Wilfrid Laurier University

6 Executive Director, KW Counselling Services, Kitchener, Ontario

7 Clinical Director, KW Counselling Services, Kitchener, Ontario

8 Director of Community Counselling Program, Family Service Thames Valley, London, Ontario

The study was conducted at KW Counselling Services, Kitchener, Ontario and Family Service Thames Valley, London, Ontario.

Word Count: 3,969 words excluding Abstract, References, Tables and Figure.

*Requests for reprints should be addressed to Carol Stalker, Faculty of Social Work, Wilfrid Laurier University, 120 Duke St. W., Kitchener, Ontario N2P2B3 Canada. E-mail: cstalker@wlu.ca

Telephone: 519-896-7585. Fax: 519-888-9732. 
WALK-IN COUNSELLING VS TRADITIONAL

A Comparison of Single-Session Walk-in Counselling and the Traditional Model for Delivering Counselling Services

\begin{abstract}
Background: Walk-in counselling has been used to reduce wait times but there are few controlled studies to compare outcomes between walk-in and the traditional model of service delivery.

Aims: To compare change in psychological distress by clients receiving services from two models of service delivery, a walk-in counselling model and a traditional counselling model involving a wait list

Method: Mixed methods sequential explanatory design including quantitative comparison of groups with one pre-test and two follow ups, and qualitative analysis of interviews with a subsample. 524 participants 16 years and older were recruited from two Family Counselling Agencies; the General Health Questionnaire assessed change in psychological distress; prior use of other mental health and instrumental services was also reported. Results: Hierarchical linear modelling revealed clients of the walk-in model improved faster and were less distressed at the 4-week follow-up compared to the traditional service delivery model. At the 10-week follow-up, both groups had improved and were similar. Participants receiving instrumental services prior to baseline improved more slowly. Qualitative interviews confirmed participants valued the accessibility of the walk-in model. Conclusions: This study improves methodologically on previous studies of walk-in counselling, an approach to service delivery that is not conducive to randomized controlled trials.
\end{abstract}


WALK-IN COUNSELLING VS TRADITIONAL

Declaration of interest: None.

Keywords: single-session therapy; walk-in counselling; outcomes; hierarchical linear modeling; mental health treatment; service delivery models; mixed methods; brief therapy 
WALK-IN COUNSELLING VS TRADITIONAL

Long waiting lists for mental health services are common. Community-based mental health and family counselling agencies have increasingly turned to the walk-in counselling (WIC) model to reduce wait times and improve accessibility. Most walk-in counselling services provide single session therapy (SST), which has been defined as "any one-visit treatment that is intended to be potentially complete unto itself" (Hoyt, 1994, p. 141). SST can be offered by appointment or within a walk-in counselling program.

No single theoretical framework guides SST; however, it is based on principles of brief therapy that encourage therapists to listen carefully to the client's goals and to emphasize the client's strengths and resources. Systemic, narrative, solution-focused and cognitive behavioral approaches are frequently used in SST (Campbell, 2012; Clements, McElheran, Hackney, \& Park, 2011; Young, 2011). Organizations offering WIC usually provide additional counselling to clients who request it or are assessed by the therapist to require it.

Although SST has been employed to cope with wait lists for many years (Denner \& Reeves, 1997; Clements et al. 2011), and a few descriptive studies of walk-in counselling services that employ SST have been published (authors, 2013), we found only one previous study of WIC with a standardized measure of clinical outcome and a comparison group (with a wait list) (Barwick et al., 2013). Children and their families using WIC had "steeper rates of improvement compared to usual care clients despite equivalence in psychosocial functioning at baseline" (p. 339).

One study of SST by appointment was found with a randomized control group and a standardized outcome measure (Perkins, 2006). Youth and their parents who attended a scheduled single session within two weeks of their request in an outpatient mental health 
WALK-IN COUNSELLING VS TRADITIONAL

clinic in Australia, were compared with controls who received the SST session after a 6week wait. Significant improvement in severity and frequency of presenting problem for the treatment group was reported compared to the control after 6 weeks. Reviews of these studies have concluded that SST may be helpful for some clients, but more rigorous research is needed (Bloom, 2001; Cameron, 2007; Campbell, 2012; authors, 2013; Hurn, 2005).

Recruitment and retention in naturalistic studies of clients seeking psychotherapy are difficult (Staines \& Cleland, 2007), but the characteristics of WIC may exacerbate this challenge. Designing studies with control or comparison groups that are similar to clients who attend WIC is difficult, in part, because of the likelihood that clients self-select when both walk-in counselling clinics and traditional service models are available. Given a choice, individuals and families who are highly distressed would be expected to attend a walk-in counselling service rather than seek services where wait lists are the norm.

We aimed to compare psychological outcomes for clients of a WIC service offering SST, with clients on a wait list for traditional counseling. We hypothesized that clients who accessed the WIC model would show faster improvement in psychological distress than those who accessed the traditional service delivery model usually involving a wait list. A second objective was to understand who may benefit most from this model.

\section{Method}

We employed a mixed methods sequential explanatory design (Ivankova, Creswell, \& Stick, 2006) collecting quantitative data in the first phase, and qualitative data in the second phase. The University Research Ethics Board approved the study. 


\section{Agency Settings}

Data were collected from two Family Counselling agencies in two urban areas located 97 kilometres apart and similar in terms of population. Family Service Agency A introduced a Walk-in Counselling Clinic (WICC) in 2007. The clinic is open one day per week from noon until 6:00 p.m. with the last client departing at approximately 8:00 p.m. The number of people attending the WICC each day ranges from 29 to $75 ; 40$ to 50 people per day is most common. Any individual, couple or family may attend without an appointment. Clients register with the receptionist and pay a fee, which is on a sliding scale geared to income ranging from no charge to $\mathrm{CA} \$ 187.50$ ( $\$ 125.00$ per hour). The intake worker who screens for risk to self or others, addictions and intimate partner violence, sees the client(s) briefly. The client(s) sees a therapist for a session normally lasting up to 90 minutes. The therapist employs a strengths-based approach that involves collaboration with the client(s) to develop a written plan. Clients are encouraged to "work the plan" and to return to the WICC (or request ongoing counselling) if needed.

At Family Service Agency B, which does not have a walk-in counselling clinic, between eight and 15 people normally telephone each business day requesting counselling, but the agency is only able to provide three to five telephone intake appointments per day. Individuals who call early enough to be included in the daily quota are given a telephone appointment with an Intake Worker, normally within a few days. The Intake Worker determines the service offered, usually placing the caller on a wait list. The Intake worker also informs callers about other community services that may be helpful to them. People calling after the daily quota has been reached are asked to call back the next business day. When given an appointment for ongoing counselling, the cost to the client is geared to 
WALK-IN COUNSELLING VS TRADITIONAL

income and ranges between no charge and CA $\$ 115.00$ per one-hour session. No other agency in that city offered WIC at that time.

Prior to data collection, we determined that clients who attended the WICC and those who requested counselling from the comparison agency were a reasonable match demographically, and the two agencies offered a similar range of other services.

\section{Recruitment Procedure}

Phase 1

At the WICC, everyone aged 16 years and older who requested service was invited by the receptionists to participate in the study, and given the Baseline Questionnaire. Research Assistants (RAs) present in the waiting room offered to assist if a client needed help to complete the questionnaire. Participants provided contact information and consent for follow-up by telephone along with the completed questionnaire.

At the comparison agency, the receptionists who received calls requesting counselling responded as usual; before terminating the call they invited callers 16 years or older to participate in the research. Several months into the study, to be sure that all clients were informed about the study, we introduced a supplementary step. The Intake Worker, when calling back in response to the initial request, was also asked to explain the study and invite the caller to participate. Callers consenting to speak with the RA were either transferred immediately to the on-site RA or asked for contact information so the RA could call them later. The RAs explained the study and the caller's rights and requested verbal consent to participate in the study. For those who consented, the RA recorded the participant's answers to the baseline questions. 
WALK-IN COUNSELLING VS TRADITIONAL

Both groups were re-interviewed by telephone four and 10 weeks after the initial questionnaire. As an expression of appreciation, participants were mailed a $\$ 10$ coffee shop gift card when they completed the 4-week follow-up telephone interviews and again after the 10 -week follow-up telephone interviews. After the 10-week follow-up, consenting participants were considered for inclusion in recruitment for the second phase of the study.

Phase 2

The qualitative component was directed by a multiple case study approach comparing two different models of service provision (Drisko, 2004, Stake, 1995, Yin, 2003). The case study sought to understand aspects of the service delivery models clients found both helpful and unhelpful. Participants were selected according to three categories with respect to their scores over time on the outcome measure (General Health Questionnaire12 (GHQ-12) (Goldberg, 1972); because the overall trend for participants from both sites was improvement on the GHQ-12, the three categories were "trend (improvement)", "nontrend (no change)" and "non-trend (deterioration)". A total of 48 interviews were conducted. Participants received another $\$ 10$ gift card when they completed the qualitative interview.

\section{Measures}

Participants provided demographic information and selected presenting problem(s) from a list of common concerns that lead adults to seek mental health counselling. The GHQ-12 is a 12-item scale developed as a self-administered screening instrument to identify psychological distress. It was designed for use in general population surveys or in clinical settings (McDowell, 2006). It has been translated into many languages and found to be reliable and valid throughout the world. It correlates highly with measures of well- 
being and other measures of distress, and has demonstrated ability to predict physician visits and psychiatric consultations. Sensitivity to change is good (Goldberg, 1972). We used the Likert-type scale scores (1-4) because they have better distributional properties for longitudinal studies of change compared to the more common GHQ scoring, developed primarily for screening purposes. Scores range from 0-36.

To measure prior use of social and mental health services, in consultation with the agency partners, we developed a measure tailored to the unique context of each agency. This measure listed local agencies providing mental health counselling and instrumental support. The latter included agencies providing assistance related to housing, employment, family violence (e.g. women's shelters) and legal issues. Participants were asked to indicate those with which they had contact and the number of visits or contacts in the previous month. They were invited to add any agencies that were not in the list. This format followed recommendations of researchers who have studied reliable ways to collect this type of data (Reid, Toban \& Shanley, 2007).

To estimate the cost-effectiveness of the two models of service delivery, participants were asked about their ability to participate in daily activities/work and about their use of formal health services in the previous month. The findings related to cost-effectiveness are reported in a separate paper.

An original semi-structured interview guide was developed for the qualitative telephone interviews, which lasted 20 to 30 minutes. The interview gathered comprehensive information about the participants, including presenting problem, nature of psychological distress, the counsellor they met with, how each service had or had not been helpful, other services used (or not) and reasons for alternative service use. It included 
open-ended questions and planned prompts to meet the needs for both structure and consistency in the interview (McCracken, 1988).

\section{Data Analysis}

Phase 1 Quantitative Data Analysis

To test the main hypothesis, we used hierarchical, longitudinal, slopes-as outcome models that used random coefficients to estimate whether the walk-in model had a differential effect on individuals' trajectories. In this hierarchical linear model (HLM; also referred to as multilevel model or mixed effects model), repeated measures were nested within participants and the effect of the walk-in model was estimated as a second level dummy variable (Singer \& Willet, 2003; Raudenbush \& Bryk, 2002). While some studies also include the clinician as a level in the HLM model, this was not feasible in this study because not all clients in the comparison group saw a clinician during the study period. We used SAS, Proc Mixed V9.2, to estimate the model by using restricted maximum likelihood (RML) estimation as recommended for multi-level models if repeated measures are not equally spaced (Singer \& Willet, 2003).

HLMs offer important advantages compared to older models, such as better handling of missing values and unequal time intervals between and within participant responses (Hedeker \& Gibbons, 1997; Nich \& Carroll, 1997). Repeated measurements also increase the statistical power, describe the shape of change over time, and avoid the psychometric problems associated with changes in scores before and after an intervention.

In developing the final model presented below, age, gender and prior service use (mental health and instrumental services) during the four weeks prior to the baseline assessment were entered into the model as control variables with the GHQ-12 score as the 
WALK-IN COUNSELLING VS TRADITIONAL

dependent variable. The age variable is centered on the average age of 35 while gender is represented as a dummy variable (Female=1; Male or Transgender=0). Prior mental health service use represents the number of reported mental health services received or attempts at accessing these services (e.g., counselling sessions or calls to a crisis service) in the month prior to baseline. While only the gender variable was significantly different between the groups at baseline, the main effects of all control variables were retained in the model for theoretical reasons. The individual trajectory is modeled as a quadratic growth model including both a linear component representing the initial slope at baseline and a quadratic component representing the curvature or acceleration in each trajectory (Raudenbush \& Bryk, 2002). Group (1=WICC) was entered as a second level dummy variable to account for the effect of the walk-in model relative to a traditional model.

Phase 2 Qualitative Analysis

Transcriptions of the telephone interviews were reviewed for accuracy before analysis began. A multiple case study approach involves analysis both within each case (model) and across cases (models) (Stake, 1995, Yin, 2003). A thematic analysis was conducted, organizing data into patterns/themes, and including a cross-thematic analysis. Coding involved five phases: becoming familiar with the data, creating initial codes, assembling codes into themes, naming and defining themes, and finally, integrating qualitative findings with quantitative findings to answer the research questions (Lofland \& Lofland, 1984). Themes were identified both inductively and deductively. Trustworthiness, credibility and verification of data were established through intercoder agreement (3 coders), lengthy and rigorous discussions identifying areas of difference to reach clarity on 
codes, memo-taking and further sampling (interviewing) (Miles \& Huberman, 1994). Themes represent repeated instances of similar responses across the data.

\section{Results}

\section{Participants}

At the WICC, out of an estimated 729 individuals who attended, 359 (49\%) completed the baseline questionnaire; 307 of the 359 (85.5\%) consented to follow-up. Of these, 221 ( $72 \%$ of those consenting to follow-up) completed data collection at the 4-week follow-up, and 229 (75\%) completed the data collection at the 10-week follow-up. At the comparison agency, out of an estimated 532 eligible individuals who requested counselling, 151 individuals (28\%) completed the baseline data collection and all of these agreed to follow-up. At 4 weeks follow-up, 146 of the 151 (97\%) completed the data collection and at 10 weeks, 142 (94\%) completed the data collection.

[Table 1 approximately here]

Table 1 illustrates demographic and other characteristics of the participants from each research site. The two samples were similar with respect to the proportion born in Canada and the proportion for which English was the first language. The participants attending the WICC were more likely to be male, and slightly younger than the comparison group. Similar proportions at both sites were employed full-time or unemployed, but those attending the WICC were more likely to report attending school and less likely to report being on either Social Assistance or the provincial disability support program.

The mean GHQ-12 score for the WICC participants at baseline was slightly higher than for the comparison group. Reported use of mental health and instrumental support 
WALK-IN COUNSELLING VS TRADITIONAL

services in the four weeks prior to baseline was similar, although the comparison agency participants reported slightly more prior contacts with other mental health organizations. Formal mental health services include visits to the Emergency Room and/or admissions to hospital for mental health reasons.

\section{Hierarchical Linear Modelling}

[Table 2 approximately here]

The estimates for the final model can be seen in Table 2. The HLM analysis confirms our hypothesis that participants in the WICC group improved faster than participants in the comparison group. There is a significant main effect of group (2.05), suggesting that, on average, participants in the WICC group were more distressed at baseline. The slope coefficient for time (-1.61) indicates that immediately after the baseline assessment, participants in the comparison group improve, on average, 1.61 points on the GHQ-12 per week. The significant interaction effect of group and time, however, suggests that the same rate for participants in the WICC group is -3.05 , almost double the rate of the comparison group. However, over time, this difference in growth rates becomes less pronounced (see Figure 1). Through a series of model comparisons it was determined that in addition to group (walk-in vs. traditional), instrumental support service use prior to baseline was related to individual changes in GHQ-12 scores over time but the interaction for age and gender was not significant. None of the three-way interactions with time, group and the control variables were significant.

[Figure 1 approximately here]

Figure 1 depicts the difference by group in GHQ-12 scores over time (Week 0-10) as predicted by the statistical model for female clients at the average age of 35 with no 
WALK-IN COUNSELLING VS TRADITIONAL

instrumental service use in the four weeks prior to baseline. These conditions were selected to represent the most typical cases. The model predicts that the WICC group participants, on average, would transition from a clinical severity level to a normal or nonclinical range (i.e., a GHQ-12 score of 13 or less) after five weeks while the average of the comparison group would not reach this clinical threshold until week 10. For the typical cases described above, the estimated effect size at four weeks is 0.24 , which is considered a small effect (Cohen, 1992). At 10 weeks, the effect size is only 0.05. Effect sizes were calculated using the pooled standard deviation of the raw scores as suggested for growth models (Feingold, 2009).

\section{Qualitative Findings}

Participants' narratives about their experiences with walk-in and/or traditional counselling helped us better understand findings from the quantitative data, in particular differences between the two models in the rate of change and for whom walk-in counselling is helpful. A participant's journey through and experience with both models of counselling was characterized in four interconnected ways. One of these, Accessibility (barriers and/or facilitators influencing a person's ability to receive and obtain services) is most salient in understanding the difference in rate of change. Being able to access service quickly and easily was very important to participants who utilized the WICC: "It was definitely something I liked. I am glad this was an option for me. From how it was before I had to keep looking to find a counsellor". Another WICC participant explained, "it was nice, because... when you have these things on your mind you kind of want to get it off right away." This stands in direct contrast to a participant attempting to access services for both herself and her adolescent daughter from the traditional model, "I mean, she was in the 
mindset to go and probably if we had been able to get an appointment that week, yes it probably would have made a difference because she was going to go, ... I can't remember exactly what transpired in that period of time (while on a wait list), but she changed her mind."

Accessibility is linked to Meaning of Service, a participant's way of making sense of their experience and understanding of the service received. The following participant explains how the ease of accessibility for the WICC helped to mobilize her and this led to a sense of self efficacy: “So I mean I have a lot of issues. I don't know if anyone can really...you know say the perfect thing that is going to make them go away ...whether it's fatigue from MS (Multiple Sclerosis) or pain from whatever or just feeling anxiety and just depression and worthlessness. I don't want to go outside ...just going out of the house that day I felt really good ... So, I'm, like, okay, I'm going to do this and I was scared as shit leaving but I felt good about it for just that. Getting that done, no, I felt a lot better after I left [the WICC]. I felt like she [counsellor] was very helpful and gave me encouragement to do things and that is kind of hard for me. So, to say that she helped me with that, that was pretty cool."

Readiness for Service, the degree to which the participant feels motivated and able to commit to and engage in counselling, helps us understand the effectiveness of the WICC and for whom it is beneficial: "It runs in my family there is a lot of people who have bipolar and a lot of depression and anxiety and I just, ... I figured I'm not going to take a chance I'm going to do it myself and I wanted to see if I could get some advice from somebody else and see if they could offer some sort of coping methods that I could utilize 
WALK-IN COUNSELLING VS TRADITIONAL

to cope when I find myself stressing out. So, I decided to walk-in and see, I guess, what I could learn and what I could apply."

\section{Discussion}

This study advances research on the WIC service delivery model by employing a comparison group, using a standardized measure, recruiting larger samples, including follow-up to 10 weeks, using HLM to analyze the quantitative data and using a mixed methods design. Another strength is the relatively high participant retention rate across the follow-up points.

The results of the HLM analysis confirm our hypothesis that participants in the WICC group improved faster than participants in the comparison group. The improvements in severity of distress are significantly different in the first few weeks following the initial contact with the agencies. Toward the end of the ten weeks, the mean GHQ-12 scores for both groups are similar even though the initial conditional mean GHQ-12 score for the WICC group was 2.05 points higher. The study supports the findings of less methodologically rigorous studies of single session therapy and walk-in counselling that reported improvement in the presenting problem following either a scheduled single session or visit to a walk-in counselling service (Authors, 2013).

Clients who were receiving more instrumental support services in the four weeks prior to requesting help at both agencies improved at a slightly slower rate than those not reporting instrumental support services at baseline. People who are receiving help with problems like housing or family violence may be less likely to improve quickly because, for example, it normally takes some period of time to find suitable housing, and the complexity 
WALK-IN COUNSELLING VS TRADITIONAL

of decisions involving family violence are well known. Prior use of instrumental support services may also be an indicator of poverty, a recognized social determinant of health.

Findings from the interviews indicate that for some clients of the walk-in model, mental health difficulties are an ongoing part of their life experience, something they need to negotiate and adapt to at different life stages. For these people, being able to have a "booster" in the form of an easily accessible walk-in session is not only helpful in terms of relieving distress, but also a good fit for those not interested in ongoing counselling. The interviews also revealed that how participants make sense of their experience and how ready they are to use counselling services may influence who benefits most from walk-in counselling.

The finding that proportionately more men accessed the WICC is similar to findings from Barwick et al.'s (2013) study of children and youth attending walk-in counselling; they reported more males were the informants for children seen in the walk-in clinic than in the usual care condition. Future research could clarify whether the walk-in counselling model is a better match than the traditional model with the help-seeking needs of some men (Evans, Frank, Oliffe \& Gregory, 2011).

Limitations of the study include the dissimilar mode of data collection between the sites at baseline (self-report vs telephone interview) and differential participation rates. Although multi-level modeling enabled us to control for the difference between sites in the mean GHQ-12 score at baseline, more similarity in terms of initial level of distress and gender would strengthen the study.

The study demonstrates that individuals attending walk-in counselling services are willing to participate in outcome research, and with sufficient resources and adequate 
WALK-IN COUNSELLING VS TRADITIONAL

training of research staff, it is possible to achieve a good response rate. More research involving multiple walk-in counselling services and additional comparison services is needed to confirm the findings of this study and to understand in more detail the essential components of an effective walk-in counselling service. 


\section{References}

Barwick, M., Uranjnik, D., Sumner, L., Cohen, S., Reid, G., Engel, K. \& Moore, J.E. (2013).

Profiles and service utilization for children accessing a mental health walk-in clinic versus usual care. Journal of Evidence-Based Social Work, 10, 338-352.

Bloom, B.L. (2001). Focused single-session psychotherapy: A review of the clinical and research literature. Brief Treatment and Crisis Intervention, 1(1), 75-86.

Cameron, C.L. (2007). Single session and walk-in psychotherapy: A descriptive account of the literature. Counselling and Psychotherapy Research, 7(4), 245-249.

Campbell, A. (2012). Single-session approaches to therapy. The Australian and New Zealand Journal of Family Therapy, 33, (1), 15-26.

Clements, R., McElheran, N., Hackney, L. \& Park, H. (2011) The Eastside Family Centre: 20 years of single session walk-in therapy. In Slive, A. \& Bobele, M. (Eds.) When one hour is all you have: Effective therapy for walk-in clients. pp. 109-127. Phoenix, AZ: Zeig, Tucker \& Theisen Inc.

Denner, S. \& Reeves, S. (1997). Single session assessment and therapy for new referrals to CMHTS. Journal of Mental Health, 6(3), 275-280.

Drisko, J. (2004). In D. Padgett (Ed.), The qualitative research experience, pp. 100-121. Belmont, California: Thomson.

Evans, J., Frank, B., Oliffe, J.L. \& Gregory, D. (2011). Health, illness, men and masculinities (HIMM): a theoretical framework for understanding men and their health. Journal of Men's Health, 8(1), 7-15.

Feingold, A. (2009). Effect sizes for growth-modeling analysis for controlled clinical trials in the same metric as for classical analysis. Psychological Methods, 14 (1), 43-53. 
WALK-IN COUNSELLING VS TRADITIONAL

Goldberg, D.P. (1972). The detection of psychological illness by questionnaire. London: Oxford University Press.

Hedeker, D. \& Gibbons, R.D. (1997). Application of random-effects pattern-mixture models for missing data in longitudinal studies. Psychological Methods, 2, 64-78.

Hoyt, M.F. (1994). Single session solutions. In M. F. Hoyt (Ed.) Constructive therapies. (pp. 140-159). London: Guilford Press.

Hurn, R. (2005). Single-session therapy: Planned success or unplanned failure? Counselling Psychology Review, 20(4), 33-40.

Ivankova, N.V., Creswell, J.W. \& Stick, S.L. (2006). Using mixed-methods sequential explanatory design: From theory to practice. Field Methods, 18(1) 3-20.

Lofland, J. \& Lofland, L.H. (1984). Analyzing social settings. Belmont CA: Wadsworth Publishing Company Inc.

McCracken, G. (1988). The long interview. Beverly Hills: Sage.McDowell, I. (2006). Measuring health

McDowell, I. (2006). Measuring health: A guide to rating scales and questionnaires. New York: Oxford University Press.

Miles, M.B. \& Huberman, A. M. (1994). Qualitative data analysis. Beverly Hills, CA: Sage.

Nich, C. \& Carroll, K. (1997). Now you see it, now you don't: a comparison of traditional versus random-effects regression models in the analysis of longitudinal follow-up data from a clinical trial. Journal of Consulting and Clinical Psychology, 65, 252-261.

Perkins, R. (2006). The effectiveness of one session of therapy using a single-session therapy approach for children and adolescents with mental health problems. Psychology and Psychotherapy: Theory, Research and Practice, 79(2), 215-227. 
Price, C. (1994). Open days: Making family therapy accessible in working class suburbs. Australian and New Zealand Journal of Family Therapy, 15(4), 191-196.

Raudenbush, S.W. \& Bryk, A.S. (2002). Hierarchical linear models: Applications and data analysis methods. Thousand Oaks, Calif: Sage.

Reid, G.I., Toban, J.I. \& Shanley, D.C. (2008) What is a mental health clinic? How to ask parents about help-seeking contacts within the mental health system. Administration and Policy in Mental Health 35, 241-249.

Shadish, W.R., Cook, T.D. \& Campbell, D.T. (2002). Experimental and quasi-experimental designs for generalized causal inference. Boston: Houghton Mifflin.

Singer, J.D. \& Willett, J.B. (2003). Applied longitudinal data analysis: Modeling change and event occurrence. New York: Oxford University Press.

Stake, R.E. (1995). The art of case study research. Thousand Oaks, California: Sage.

Staines, G.L. \& Cleland, C.M. (2007). Bias in meta-analytic estimates of the absolute efficacy of psychotherapy. Review of General Psychology, 11(4) 329-347.

Yin, R.K. (2003). Case study research ( $3^{\text {rd }}$ ed.). Thousand Oaks, California: Sage.

Young, K. (2011). Narrative practices at a walk-in therapy clinic. In Slive, A. \& Bobele, M. (Eds.) When one hour is all you have: Effective therapy for walk-in clients. pp. 149166. Phoenix, AZ: Zeig, Tucker \& Theisen Inc. 


\section{Figure 1}

Estimated GHQ-12 Scores for Each Group Over Time (for 35 year old female clients with no prior service use)

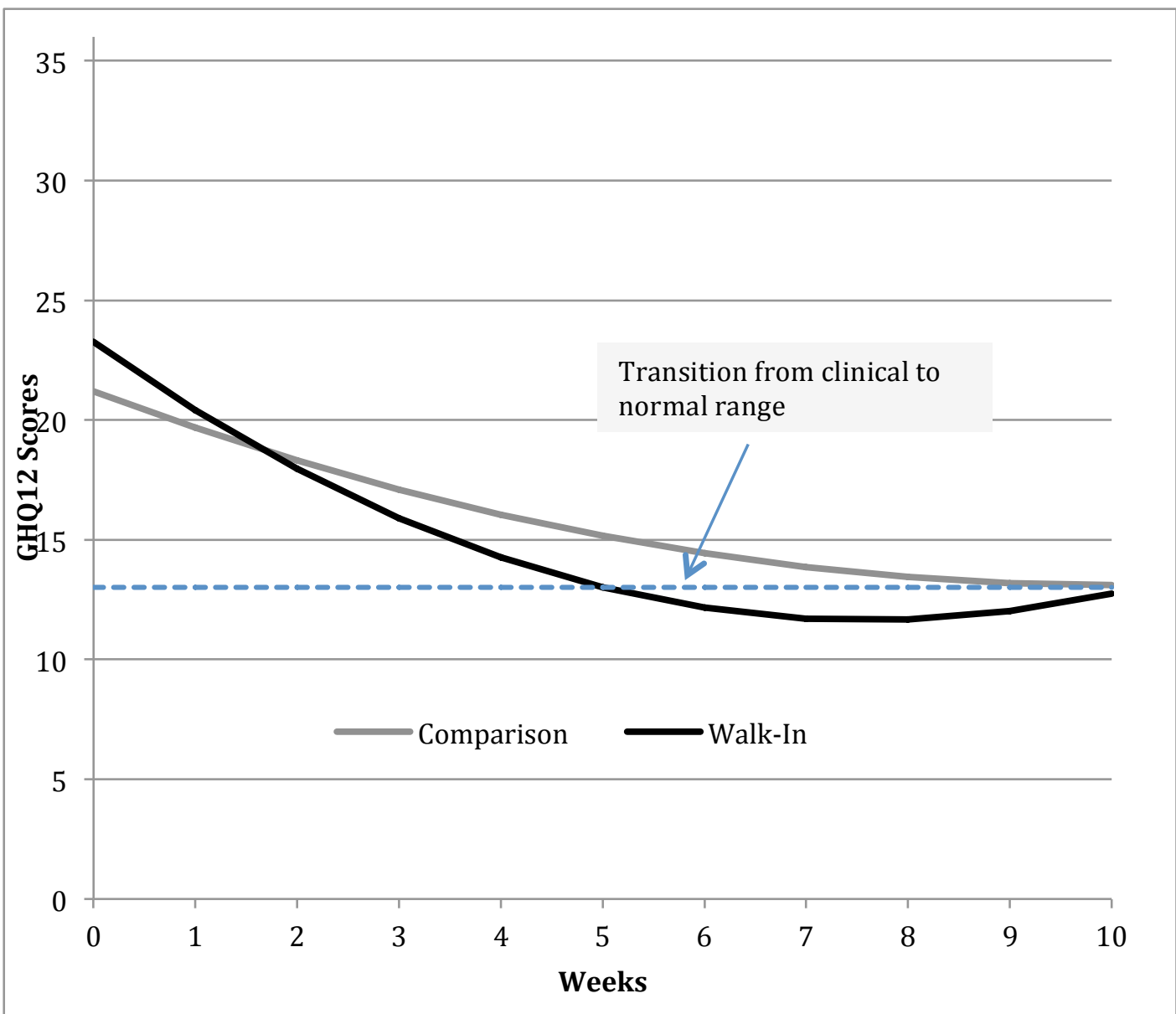

